\title{
ITINERARIOS PROFESIONALES DE VIOLONCHELISTAS: UN ESTUDIO BIOGRÁFICO NARRATIVO
}

\author{
Marina Sanchis López \\ Conservatorio Profesional de Música de Torrent \\ marsanlop.07@gmail.com
}

\begin{abstract}
La mayoría de los esfuerzos en los últimos años para mejorar la eficiencia y la responsabilidad en las reformas curriculares del aprendizaje profesional instrumental en los conservatorios españoles, se ha realizado desde las perspectivas del estudiante y el docente, la adecuación pedagógica y las circunstancias políticas. Sin embargo, apenas aparece explorada la perspectiva del profesional instrumental con itinerarios dispares. Para poder configurar propuestas formativas afines a la realidad laboral existente se hace necesario también comprender esta dimensión.
\end{abstract}

Most of the efforts in recent years to improve efficiency and accountability in the curriculum reforms of the professional instrumental learning in Spanish conservatories, have been carried out from the perspectives of the student and the teacher, the pedagogical suitability and the political circumstances. However, the viewpoints of the instrumental practitioner with diverse itineraries appears barely explored. To configure training programs according to the work reality, it is also necessary to understand this dimension.

\section{INTRODUCCIÓN}

El presente artículo se encamina a conocer y describir los problemas, necesidades y demandas del colectivo de violonchelistas profesionales, mediante un enfoque biográfico-narrativo. Constituye un trabajo con técnicas de investigación cualitativa que analiza el desarrollo profesional de una muestra de tres violonchelistas profesionales en el contexto español actual. Para ello, se ha tenido en cuenta la dimensión que aportan los estudios que cubren la perspectiva histórica del violonchelo en profundidad, comprendiendo en qué manera la historia ha examinado las vidas de nuestros predecesores violonchelistas. Así pues, comparto la opinión de Walden (1998) y Stowell (1999) de que el contacto habitual con los violonchelistas de antaño, hasta más o menos 1850, han sido las piezas, los ejercicios y los métodos de aprendizaje enseñados a través de generaciones de violonchelistas, y de que todos estos trabajos, incluso con sus reediciones, nos indican poco sobre las características performativas de sus autores y nada sobre las cualidades de la persona que las creó.

Es a partir de la creación del Conservatoire National de Musique et de Déclamation de París en 1795 cuando, paulatinamente, además de estos trabajos se empiezan a recoger informaciones particulares sobre músicos individuales, su forma de interpretar, y también sobre las famosas escuelas nacionales de interpretación. A partir de este momento, los violonchelistas cobran una cierta estabilidad social, se codifican las técnicas de interpretación y de construcción del instrumento y el arco, y se multiplican las publicaciones musicales.

Afortunadamente, la revisión y análisis desde una perspectiva científica por parte de chelistas o investigadores contemporáneos no sólo de estos ejercicios y composiciones, sino de las vidas de nuestros ascendientes desde cualquier perspectiva, nos ayuda a apreciar la herencia cultural que nos han dejado y, sobre todo, a comparar los elementos de las carreras profesionales que han cambiado con el paso del tiempo, así como aquellos que se mantienen intactos. De esta manera, existen trabajos centrados en estudiar las composiciones de algunos de ellos (Dorsam, 2006; Pezzoli, 2011), en conocer la figura y detalles de un único chelista (Park, 2004), o en establecer las influencias de las diferentes escuelas de violonchelo en el mundo a través de entrevistas personales a violonchelistas (Gagnon, 2006).

En el momento en que se plantea esta investigación, el contexto educativo europeo se encuentra inmerso en una reforma referida a la Educación Superior. En España, la Ley Orgánica 2/2006, de 3 de mayo, de Educación, regula de forma específica, en sus artículos 54 a 58, las enseñanzas 
artísticas superiores, señalando como tales los estudios superiores de música entre otros. La definición del contenido de estas enseñanzas musicales, así como la evaluación de las mismas, según el artículo 46 de la citada ley, se hará en el contexto de la ordenación de la educación superior española y en el marco europeo. Este hecho supone un esfuerzo y un paso de gigante por parte de todo el mecanismo administrativo y docente, puesto que los centros superiores deben ofertar un nuevo modelo de estudios dirigidos a la obtención del título de graduado o graduada. Aplicar nuevas metodologías de aprendizaje adaptándose a nuevos modelos educativos afecta, además, a las enseñanzas elementales y profesionales que deben proporcionar al alumno una formación artística de calidad formando, orientando y preparando al alumno para poder cursar las nuevas enseñanzas superiores y, más adelante, acceder al mercado laboral con garantía de éxito.

Por otra parte, este giro en la educación superior afectará en pocos años a la reestructuración del mercado laboral de cualquier profesional de la música, el cual probablemente deberá adaptarse a otras condiciones y exigencias. Inmersos en este proceso de cambios que afecta a todos los niveles educativos, se hace necesario escuchar y revisar detenidamente no sólo la voz del docente, sino la de cualquier profesional de la música de la misma especialidad que no tenga como itinerario laboral la docencia - profesores de orquesta, intérpretes autónomos- con el fin de aportar conocimiento de la realidad actual y competencia para asumir los cambios que se están produciendo.

Desde la perspectiva de un estudio detallado de las vidas de tres violonchelistas, se espera poder dar al lector de este trabajo un esbozo significativo del panorama actual del violonchelo en España. Tomando como aval la revisión bibliográfica que se refiere al concepto de desarrollo profesional (Bolívar, 1999; Buendía Eisman, 1998; Hookey, 2001), se sostiene que este tipo de reflexión sobre la propia historia profesional es una perspectiva valiosa de conocimiento personal y profesional. Para ello se ha planteado un esquema conceptual general de objetivos para analizar:

- Conocer las trayectorias formativas y profesionales de una muestra de chelistas que siguen itinerarios profesionales diferentes.

- Identificar cuáles son los aspectos más relevantes de esas trayectorias y que influyen en el desarrollo de su profesión.

- Identificar los elementos personales y culturales que a lo largo de una vida configuran el saber profesional de un chelista.

\section{MARCO TEÓRICO}

Para la ubicación teórica del artículo se han realizado lecturas referidas al modelo biográficonarrativo en ciencias sociales, sobre todo las que abordan procesos metodológicos (Cantón, 1996; Denzin, 1970; Pujadas, 1992), que han aportado la idea de la emergencia de saberes personales implícitos.

Aunque las que sin duda han encauzado este trabajo han sido las especializadas en educación musical, como las que apuntan a los diferentes contextos educativos y sus interacciones para mejorar en qué y cómo se enseña (Bresler, 2004), así como las que indican la investigación en educación musical desde una perspectiva histórica (Cox, 2006), y aquellas que han aportado modelos de investigación que permiten conocer las realidades de los distintos profesionales (Ocaña, 2006a, 2006b) y resto de la comunidad educativa (Burnard, 2005; Cain, 2005) para así configurar futuras propuestas formativas más afines a la realidad educativa.

A partir de este momento, se ha centrado la atención en los numerosos estudios sobre desarrollo profesional y perfiles profesionales, fundamentalmente, en el contexto educativo (Bolívar, 1999; Butt, Towsend y Raymond, 1992; Fernández Cruz, 1998; Kelchtermans, 1993; Kelchtermans y Vandenberghe, 1994; Knowels, 2004; Laffitte, 1991). Todos ellos aportan a la perspectiva de 
desarrollo profesional la idea de un continuo cambio y aprendizaje tanto en el plano personal como en el profesional, incluso cambios en los contextos político, social y económico.

Por otra parte, debido a la naturaleza exploratoria del estudio, se ha hecho necesario consultar la dimensión referida a la educación musical general -en la cual se inscriben la enseñanza y aprendizaje del violonchelo-, aportando marcos teóricos que nos ayudan a comprender el desarrollo de procesos que implican el aprendizaje musical del alumno (Bresler, 2007; Colwell, 2007; Rusinek, 2004, 2006).

Aunque sin descuidar la dimensión referida a interpretación musical -que constituye parte importante del desarrollo profesional de un chelista-, que ha aportado consejos prácticos y principios y procedimientos que subyacen en este proceso (Jørgensen \& Lehmann, 1997; Rink, 1995; Rink, 2002), se ha continuado con el enfoque del contexto docente, de manera general, porque una parte del desarrollo profesional de un chelista tiene que ver con la enseñanza -aunque no se dedique plenamente a ella-, y porque no se encontraron estudios previos a éste que hicieran referencia a perfiles y desarrollos profesionales del colectivo de violonchelistas de manera global en España.

Sin embargo, se referencian dos artículos, uno referido a perfiles y profesiones de la música en España que correlaciona las profesiones con los estudios musicales que se pueden cursar (Cañada, 2008), y otro de Polo (2008), violonchelista que intenta diagnosticar desde una perspectiva personal los diferentes problemas que surgen en torno a la experiencia docente que realiza en el País Vasco. Especialmente interesantes son sus reflexiones sobre qué significa ser un instrumentista profesional hoy en día. Además, se ha explorado la dimensión específica referida a enseñanza y aprendizaje instrumental (Hallam, 1998), aportando conocimiento y pensamiento actual sobre la enseñanza y aprendizaje instrumental efectivos de manera general, independientemente del instrumento.

\section{DISEÑO Y DESARROLLO DE LA INVESTIGACIÓN}

\section{Enfoque metodológico}

En relación lógica al paradigma cualitativo y constructivista que se ha tomado como referencia, se han utilizado métodos y técnicas biográfico-narrativas, las cuales permiten la emergencia de saberes personales implícitos, a veces no reconocidos. Una de las consideraciones principales a tener en cuenta cuando hablamos del método biográfico es la comprensión desde diversos ángulos del término historias de vida. Según Bolívar (1999, p. 176) "si bien los relatos de vida siempre hacen referencia a la singularidad de una vida, refleja a la colectividad social de que se trate". Esto conduce a un respeto por la naturaleza del conocimiento y las acciones de los profesionales de los que trata este trabajo y a la correspondiente necesidad de personas ajenas o externas a él de comprenderlos.

\section{Estrategia de recogida de información}

He utilizado como estrategia de recogida de información la entrevista biográfica siguiendo las orientaciones planteadas por Bolívar (1999) sobre el desarrollo profesional y los ciclos de vida del docente de secundaria, utilizando el método de incidentes críticos (Burnard, 2005) como técnica para inducir a la reflexión a los entrevistados. Para no desviarme durante las entrevistas he establecido un patrón de curvas (cada curva es un incidente crítico en la vida musical), que se agrupa en los siguientes bloques:

1. Etapa inicial: elección de los estudios musicales y del violonchelo en concreto.

2. Trayectoria formativa.

3. Transición al mundo laboral.

4. Trayectoria profesional. 
Cada entrevista ha durado entre dos y tres horas, ha sido grabada, y posteriormente transcrita. Las transcripciones han sido revisadas por los mismos interlocutores para así poder añadir o vetar información. Una vez completada la revisión, he comenzado la fase de análisis para ver qué datos emergen. He evitado plantear preguntas que se contestaran con un sí o con un no y las sesiones se han realizado siguiendo un orden cronológico para facilitar una buena disposición del recuerdo de los diferentes momentos vividos. Durante las sesiones se han producido saltos cronológicos aunque no se interrumpió a ninguno de los interlocutores para no segar el desarrollo de algún tema a través del tiempo.

\section{Criterios de selección y características de los informantes}

Para encontrar informantes fiables que nos ayudaran a comprender y contextualizar los objetivos presentados al inicio del estudio, se imponía la búsqueda de una muestra lo suficientemente diversa de chelistas tanto en su formación como en sus itinerarios profesionales. Me interesaban tres informantes tanto por lo que tienen de único cada uno de ellos como por lo que tienen en común, e insisto en la necesidad de conocer todo lo que implica en conocimiento de cada uno de los informantes, sus temas y sus complejidades (Stake, 1998). Esto me ha dado una visión amplia del campo profesional y así he podido reflexionar sobre el impacto de sus transiciones evolutivas y establecer puntos coincidentes y divergentes en sus diferentes trayectorias.

Los criterios de selección de una muestra de tres violonchelistas profesionales son los siguientes:

- Tener entre 35 y 45 años

- Cumplir al menos 10 años en el ejercicio de la profesión

- Desarrollar su labor como violonchelistas en distintos itinerarios profesionales:

- Docente en conservatorio

- Profesor de orquesta

- Intérprete autónomo

- Tipo de formación

- Reglada

- No reglada

Las dos primeras variables están relacionadas entre sí. Se precisaban informantes que tuvieran unos años de experiencia laboral cumplidos y una formación muy extensa, por lo tanto quedaban excluidos los menores de 35 años y los mayores de 65 -son más expertos pero carecen en general de una formación extensa moderna y su etapa de transición al mundo laboral es lejana en el tiempo.

Las dos siguientes variables son también vitales, ya que se hacía necesario encontrar informantes con trayectorias diferenciadas que nos ayudaran a comprender y contextualizar los objetivos presentados al inicio del estudio. Tal como aparece en el cuadro-resumen siguiente detallo las características de cada informante con su correspondiente pseudónimo:

\begin{tabular}{|c|c|c|c|c|}
\hline Pseudónimo & Itinerario profesional & Tipo de formación & $\begin{array}{c}\text { Años en el } \\
\text { ejercicio de su } \\
\text { profesión }\end{array}$ \\
\hline C1 & Enseñanza en un centro oficial & Reglada (en España) & 45 & 17 \\
\hline C2 & Orquesta profesional & $\begin{array}{c}\text { No reglada (en España) y reglada } \\
\text { (en el extranjero) }\end{array}$ & 40 & 13 \\
\hline C3 & Trabajo autónomo & Reglada (en España y el extranjero) & 41 & 14 \\
\hline
\end{tabular}




\section{Criterios de calidad de la investigación}

Prácticamente desde el inicio de la investigación han sido constantes mis reflexiones referidas a los criterios más apropiados para juzgar las contribuciones de este estudio desde el enfoque cualitativo. El procedimiento que he utilizado para hacer válidos los criterios de esta investigación es la triangulación, que es la convergencia de múltiples fuentes de evidencia o la reiteración de apelaciones que amparan las conclusiones (Eisner, 1998). Revisados los trabajos de Stake (1998) y Cantón (1996), he utilizado los siguientes métodos de triangulación:

- Triangulación de la información o de las fuentes de datos: Implica comprobar la autenticidad de aquello que se cuenta a través de otras fuentes escritas de datos. La información obtenida en las entrevistas ha sido contrastada con la legislación educativa y la bibliografía especializada sobre el violonchelo y su enseñanza, además de aquella referida a la educación musical general.

- Triangulación interna o del investigador: Implica la revisión del mismo fenómeno por parte de uno o varios investigadores, observadores y/o actores. En este proceso de indagación después de haber realizado las entrevistas, éstas han sido transcritas, interpretadas y enviadas a cada informante para su revisión. Me ha sorprendido que los tres entrevistados apenas hicieran cambios ni sobre las ideas expresadas ni por el lenguaje espontáneo que desprende cada una de la sesiones. Los únicos juicios que alguno de ellos ha pedido que se modificaran, son los referidos al anonimato de terceras personas que han surgido durante las conversaciones.

- Triangulación de la teoría: Implica la revisión de literatura científica buscando teorías que nos sirvan para interpretar y aclarar diversos aspectos de la información recogida. La revisión de la teoría me ha permitido profundizar en el problema de estudio surgiendo así los diferentes temas planteados al inicio del estudio.

\section{Procedimiento de análisis}

Esta fase de la investigación -dado que el estudio es en esencia cualitativo- ha exigido extrema organización en la disposición de la información y datos obtenidos de las entrevistas. Así pues, el proceso de análisis se inicia ya con la revisión de la literatura afín para realizar el marco teórico de la investigación, además de encuentros con los chelistas con los que me socialicé previamente sin abandonar la revisión constante de los objetivos iniciales. A partir de aquí, organicé y reduje datos con el fin de crear un protocolo de entrevista semi estructurada y crear un sistema de categorías inicial. Así, a partir de una masa desordenada de datos, inicié una secuencia coherente de información con la que comenzar. Durante las sesiones de las tres entrevistas, la transcripción y primeras revisiones de las mismas continué refinando este sistema centrándome en las experiencias de los entrevistados que eran más relevantes para la investigación.

Miles y Huberman (1994) presenta un esquema de análisis que he tomado como modelo y que supone tres fases -reducción de la información, disposición y transformación de la información y extracción de conclusiones y verificación. El proceso por tanto, una vez organizada la información de las entrevistas en segmentos para hacerla manejable y operativa desde un criterio espacial, comienza clasificando conceptualmente las unidades que son cubiertas por un mismo tópico con significado-categorización. Inmediatamente después se les ha asignado un código propio de su categoría. Estos códigos son palabras con las que se van etiquetando las categorías. Las categorías se han ido renombrando durante todo el proceso al utilizar un criterio mixto de categorización inductivo-deductivo.

Etapa inicial:

- Contexto sociocultural y educativo

- Contexto familiar

- Elección

- Formación inicial 
Etapa formativa:

- Elección de profesorado

- Tipo enseñanza

- Influencias contexto educativo

- Valoración de la formación

Transición al mundo laboral:

- Elección itinerario

- Paso al mundo laboral

Trayectoria profesional:

- Práctica profesional

- Elementos personales

- Vida familiar

A partir de este momento utilicé la reconstrucción de vida como documento principal en el que interpreto y analizo la información de las entrevistas biográficas siguiendo el modelo de incidentes críticos (Bolívar, 1999, pp. 183-188; Burnard, 2005) dentro de un criterio cronológico, que permite una mejor ubicación y organización de la información, analizando así pasado, presente y futuro.

Además elaboré también unos biogramas de la trayectoria profesional de cada chelista en los que analizo verticalmente la información de una forma más clara centrándome en los elementos más significativos que han afectado a su desarrollo profesional incluyendo las categorías de:

- Cronología

- Categorías

- $\quad$ Experiencias y condicionantes

- Elementos que inciden en el desarrollo profesional

\section{RESULTADOS}

A continuación, una vez comparadas las tres historias de los violonchelistas entrevistados, recojo un análisis de las experiencias e influencias que han generado y contribuido a construir el conocimiento de su profesión y una identificación de los aspectos más relevantes de esas trayectorias y que influyen en el desarrollo de la misma, tal como se plantea en los objetivos al principio del artículo. Este proceso es subjetivo, puesto que ofrezco mis propias construcciones de las construcciones de las personas a las que he estudiado y únicamente pretendo describir una realidad que responde a los objetivos iniciales del trabajo.

\section{Aspectos relevantes de las trayectorias profesionales}

En líneas generales las experiencias infantiles, los padres y la familia, los primeros profesores y el entorno cultural de los tres entrevistados conforman saberes iniciales adquiridos. Se conecta aquí con las ideas expresadas por Kelchtermans (1993) y por Kelchtermans y Vandenberghe (1994) sobre la perspectiva biográfica.

En contraposición a otras profesiones en las que el contacto inicial coincide con el inicio de su formación profesional, todo músico dedicado a la práctica instrumental y la docencia comienza a formarse en las estrategias y modelos de su profesión desde su infancia, y continúa viviéndolas durante todo su proceso formativo hasta alcanzar la madurez en su entorno laboral. Todo esto se debe a que los elementos que actualmente manejan en su práctica profesional los han vivido desde los inicios de su educación musical e instrumental (Polo, 2008). 
C2: [...] Ya los ejercicios que me da, como por ejemplo poner la mano plana y calentar...tonterías que eran para críos pero que venían muy bien...guardar las líneas de los brazos...ejercicios que no sé si entonces estaban ya publicados... [...]

Además, considero determinante la diferencia y los aspectos particulares de cada caso tales como: la idea de pensamiento especialista que se inculca a uno de los entrevistados como chelista orquestal ya desde muy pequeño y que no adquieren sus compañeros. No sólo es iniciado en la práctica instrumental, sino que es introducido en las estrategias y modelos de violonchelista orquestal desde la infancia, y esto se manifiesta como pieza fundamental en el conocimiento de la especialización de su futuro itinerario laboral.

$\mathrm{C} 2:[\ldots] \ldots$ pienso en que todos mis profesores han sido chelos de orquesta. Recuerdo mi primer encuentro con [profesor de violonchelo 1], me compra un chelo de $3 / 4$ acompañado de mi padre. [...]

En lo que se refiere a la relación maestro-alumno instrumental se revela como individual y única. Aunque existen métodos y tratados en los que se describe minuciosamente el saber hacer de cada escuela violonchelística, la fuente de conocimiento principal es la persona que aglutina todos esos conocimientos, que a su vez aprendió de otra persona singular y única (Walden, 1998). El conocimiento y la técnica instrumental se han transmitido y evolucionado durante generaciones de chelistas de esta manera. Así pues, se aprecia cómo las destrezas adquiridas como alumnos, el momento y la manera en que las adquieren, condicionan parte de la elección de itinerario y el desarrollo profesional de los tres chelistas.

C1: [Profesor de violonchelo 3] me dijo que fuera a estudiar a [ciudad española 2] con él y así fue. Me fui con él y empezó una nueva vida muy diferente. En ese momento él tenía en torno a cuarenta y pocos años y fue una persona que me ayudó mucho porque creyó en mí lo primero y yo me fui allí y nunca me cobró una clase, se ocupó de mí y...bueno hay gente que no opina lo mismo de él, pero yo siempre digo que es una persona a la que le debo haber pasado de un chaval de pueblo que estaba más despistado a de repente empezar a estudiar, hacer escalas, estudios...etc. Con su manera poco ortodoxa, a lo mejor. Pero una persona que en aquel momento conmigo se volcó y a mí me parecía fabuloso trabajar con una persona que todavía tenía fresco a Tortelier, Fournier o Navarra, que bueno, que uno estará más o menos de acuerdo con ellos, pero no dejan de ser gente de altura. Trabajé con él muchos años y me enseñó una manera de hacer, que luego lógicamente fue evolucionando.

Sus diferentes profesores han marcado claramente la actitud, modelos y creencias sobre los que se posicionan los tres en el ejercicio de su profesión. En el caso de las influencias positivas, el discurso de los tres coincide en aquellas en las que reflexionan sobre el saber hacer, el aprendizaje de una metodología y una técnica básica, que naturalmente continua evolucionando a lo largo de toda su carrera.

C1: En el caso de [profesor de violonchelo 4] lo tenía todo organizado y él fue la persona que hizo que sistematizara muchas cosas, más que en el cuerpo en la mente. De repente yo vi que ahí había un camino y una manera de hacer y unos conceptos musicales y técnicos, que puedes estar de acuerdo con él o no, pero infalible.

C2: [...]...y él decía "esto hay que limpiarlo. Tienes un motor que no funciona. Vamos a quitar pieza por pieza y vamos a limpiarlo y montarlo de nuevo y cuando eso esté veremos qué pasa". Esa sensación de...era otra filosofía que me sirvió porque me enseñó a hacer cambios de posición.

Entre las influencias negativas destacan la incorrecta adquisición de técnica básica, y la gestión del cuerpo y repertorio básico. Aquí rechazan un modelo de profesional que conoce muy bien los elementos de su profesión puesto que los maneja día a día pero que no sabe extrapolarlos y 
transmitirlos desde una perspectiva integral a sus alumnos sincronizando con las ideas expresadas por Hallam (1998) respecto a las habilidades a desarrollar en la dimensión interpretativa.

Para los tres, otra de las fuentes de conocimiento principal de su profesión ha sido el marcado carácter experiencial al que se han tenido que someter durante su período formativo, a diferencia de otras profesiones en las que la fuente de conocimiento principal es de marcado carácter teórico. En este punto, coinciden todos en que su paso por el conservatorio o las escuelas de música en España -condicionado siempre a que hubiera un buen profesor de instrumento-, les ha proporcionado los conocimientos técnicos y musicales necesarios para su profesión pero no la experiencia ni los modelos profesionales que siguen en su itinerario profesional actual. Uno de ellos la ha adquirido a través de experiencias profesionales tempranas. Los otros dos entrevistados han adquirido esta experiencia en instituciones en el extranjero de reconocido prestigio, donde el proyecto educativo está perfectamente integrado en la realidad laboral de su contexto y en continua evolución.

C3: [...] Teníamos un ciclo de Música de Cámara en el [sala de conciertos 1] que era tocar mezclados con gente de la Sinfónica de [ciudad americana 1], era otra vez tocar el Octeto de Schubert, Sextetos de Brahms...salíamos en la radio y ganábamos dinero y estaba muy bien.

C2: [...] Tengo que darle las gracias a la institución porque era una facultad, como facultad me refiero a que había un criterio musical y técnico generalizado en todo el departamento de cuerda y en otros departamentos. Te daban la sensación en música de lo que estaba bien y de lo que no lo estaba, de lo que era presentable y de lo que no lo era. Tenía profesores de cámara que hablaban el mismo lenguaje que mi profesor de chelo.

Esta situación se debe a que en España, el plan de estudios de 1966 en el que las asignaturas podían ser distribuidas de manera aleatoria durante todo el grado medio y el grado superior tanto si se cursaban de manera oficial como si se accedía como alumno libre desfavorecía el ambiente musical extraescolar. La enseñanza musical reglada se reducía a la clase principal de instrumento en la que se prestaba mucha atención a los ejercicios técnicos, y a un conjunto de asignaturas que distribuidas temporalmente de manera aleatoria no favorecían el aprendizaje integral de la música desde diferentes ángulos. Así, dos de los entrevistados, se decantan e incluso describen de manera minuciosa, una vez finalizada su enseñanza reglada en España, los centros educativos extranjeros donde continúan su formación. Estos gozan de una clase de violonchelo internacional, y de un ambiente musical extraescolar extraordinario en el que reciben formación camerística y orquestal muy sólida con programas de colaboración e inmersión laboral con orquestas profesionales y festivales de música de cámara de prestigio. Otro de los entrevistados, por el contrario no decide continuar sus estudios en el extranjero, adquiere todas estas influencias fuera del contexto educativo a través de la experiencia vivida en sus primeros contactos con el mundo laboral y a través de su último profesor de instrumento, que aúna conocimientos técnicos, interpretativos y culturales.

También los entrevistados hacen referencia a experiencias vividas en jóvenes orquestas, concursos, festivales, actividades extraescolares y a vínculos de amistad que en su vida profesional se convierten en vínculos laborales. Cada una de estas experiencias se convierten en micro puntos de inflexión a partir de los cuales comienzan a configurar sus creencias sobre su futura profesión, a posicionarse sobre los futuros itinerarios laborales y a tomar decisiones sobre su propia línea educativa, cambiando de rumbo cada vez que lo consideran necesario.

C1: Desde el punto de vista de ser un estudiante comencé a ser un profesional aunque en una orquesta con faena discontinua y en aquella época había muchos conciertos al año y viajes. Yo pasé de llegar allí con mi chelo, mis estudios y mi repertorio a... yo no había hecho más orquesta que la de cámara de [ciudad natal] y con una perspectiva también complicada. Recuerdo que entré en ese momento y había giras. [... ]

Cuando valoran y reflexionan sobre su formación extrapolan este pensamiento a una reflexión crítica sobre su profesión. Los tres abogan por una educación musical y violonchelística más integral donde el profesor de instrumento aúne no sólo conocimientos técnicos, sino interpretativos 
y culturales, y que las instituciones españolas sigan esta misma línea fomentando un ambiente extraescolar donde se adquieran experiencias musicales fuera de las aulas aunque dentro del contexto educativo, y estableciendo lazos con la práctica profesional real en el caso de la educación superior.

$\mathrm{C} 1:[\ldots]$ El intérprete es un intérprete, igual que el pintor es pintor, quien hace Bellas Artes no es un pintor. Tú no puedes formar un pintor simplemente porque vaya a la facultad y haga Bellas Artes. No puede ser, hay mucho más detrás de eso, y aquí habrá que buscar la manera de mejorar. La postura oficial no es muy halagüeña pero yo creo que la experiencia de cada uno de nosotros nos tiene que enseñar que hay que encontrar un camino. [... ]

\section{Elementos personales y culturales}

Respecto a los elementos personales y culturales que configuran el saber profesional de esta muestra de tres violonchelistas, en líneas generales se observan algunos rasgos personales y culturales en común que los definen.

El hecho que más llama la atención, es el sentimiento de pertenencia a un colectivo -el de violonchelistas en general. Podemos destacar además que apenas aparece difuminado el de pertenencia a una especialidad como en otras profesiones -orquesta, docencia, música de cámara, autónomo. Sobre todo el hecho de que el rol referido al ejercicio como violonchelista autónomo no tenga una alta consideración en la sociedad española debido a la inexistencia de un mercado de intérpretes autónomos estable, hace que no emprendan proyectos nuevos siguiendo esta línea. Todos coinciden en que la cultura de violonchelista autónomo está plenamente consolidada en Europa y los tres desde su puesto reivindican y luchan porque se instaure y consolide en España. Además, abogan por una educación integral que pueda abrir a los futuros profesionales campos de especialización más concretos y que ellos no gozaron en España en su momento. Este hecho está corroborado porque en los conservatorios españoles existe la figura del profesor de violonchelo y no una figura especializada en música de cámara, repertorio orquestal o pedagogía de violonchelo hasta bien entradas las enseñanzas superiores. En los profesionales en concreto, no existe además ninguna asignatura en los últimos cursos de enseñanzas profesionales que suponga una iniciación a cualquiera de estas especializaciones. La única vía es que el profesor mismo de violonchelo proponga la creación de una optativa (que únicamente puede cursarse durante un año) referida a la iniciación en repertorio orquestal, camerístico o de pedagogía del violonchelo.

C1: La profesión en España es complicada. Porque tú tampoco tienes seguro...que un intérprete tenga una actividad de conciertos regulares más o menos -algunos meses más que otros- y estar siempre pensando en los que tocarás el próximo mes....es imposible. Yo desde los 19 años hasta ahora me he mantenido así y no deseo nada más pero cada vez es más complicado. [... ]

Todos señalan que decantarse por la especialización en su educación y más tarde en el itinerario profesional, aunque algunas veces no fuera vocacional, ha constituido un éxito a nivel laboral. A unos les ha proporcionado estabilidad $\mathrm{y}$ a otros la posibilidad de seguir mejorando $\mathrm{y}$ promocionando.

C2: [...]... pienso en que todos mis profesores han sido chelos de orquesta. Recuerdo mi primer encuentro con [profesor de violonchelo 1], me compra un chelo de $3 / 4$ acompañado de mi padre. [...]

Otro de los aspectos que destaco es que los tres son violonchelistas muy preocupados por la reflexión de quehaceres prácticos, pero no se quedan ahí y la extrapolan a una reflexión crítica sobre su profesión. Esto fomenta y dinamiza la mejora de su propia práctica profesional y la de su colectivo porque siempre aportan aspectos nuevos, que adecuadamente difundidos pueden mejorar la realidad laboral.

C2: [...] Estás en un colectivo del que es importante saber si quieres hacer música con él. Para hacer música con una persona tienes que saber algo de ella. Esto también lo aprendí de mayor. [...]

C3: Todas las cosas que se dicen en el ensayo tienen que ser muy profesionales, el ego se deja aparte. No puedes imponer tu forma de tocar sino que tienes que ayudar a que la música en conjunto aflore. Así también aprendes de tus colegas estilos y formas de tocar nuevas. Me alegro de aprender de ellos la inspiración para poder hacer la música diferente. 


\section{Sugerencias}

En primer lugar, como continuación de este estudio piloto, sería recomendable elevar el número de violonchelistas entrevistados para poder así profundizar por ejemplo en el desarrollo profesional de este colectivo de manera más general. De esta manera se podrían establecer sus ciclos de desarrollo profesional y las necesidades y demandas de este colectivo con sus correspondientes itinerarios laborales.

Este estudio también puede servir de base a líneas de investigación dirigidas a la reconfiguración de los diferentes contextos educacionales. Entre los conservatorios y las instituciones deben propiciarse diferentes colaboraciones profesionales e interdisciplinarias para poder educar primero y fomentar la inserción laboral después. En los últimos años se habla mucho acerca de trabajar para la calidad y que es prioritaria. Este concepto se concibe desde un enfoque técnico, como si los alumnos fueran la producción de una fábrica y sus profesores las máquinas. Esta investigación sugiere un carácter más colaborativo y colectivo en la concepción de este término. Para analizar aquello que no funciona es necesario realizar proyectos de cambio, partiendo de las necesidades reales del profesor, del intérprete autónomo y del profesor de orquesta. Es importante tomar en consideración la experiencia profesional y personal de los diferentes profesionales, además del contexto y del alumnado.

En segundo lugar, independientemente de los diseños de currículos oficiales -expuestos la mayoría de las veces al clima político dominante- es necesario que en nuestra práctica diaria tengamos un esquema docente claro que garantice a los alumnos que pasan por el conservatorio la adquisición de competencias para desarrollar su futura profesión en la especialización que sea.

\section{Referencias}

Bolívar, A. (Ed.). (1999). Ciclo de vida profesional del profesor de secundaria. Desarrollo profesional y formación. Bilbao: Mensajero.

Bresler, L. (2004). Metodología de investigación cualitativa: Prestando atención a la música escolar como género en sus micro y macro contextos Revista Electrónica Complutense de Investigación en Educación Musical, 1(1). Consultado en http://revistas.ucm.es/index.php/ RECI/article/view/RECI0404110001B

Bresler, L. (Ed.). (2007). International handbook of research in arts education. Nueva York: Springer.

Buendía Eisman, L. (1998). El proceso de investigación. En M. P. C. Bravo (Ed.), Investigación educativa (pp. 69-107). Sevilla: Alfar.

Burnard, P. (2005). El uso del mapa de incidentes críticos y la narración para reflexionar sobre el aprendizaje musical. Revista Electrónica Complutense de Investigación en Educación Musical, 2(2), 1-15. Consultado en http://revistas.ucm.es/index.php/RECI/article/view/9583

Butt, R., Towsend, D., \& Raymond, D. (1992). El uso de las historias de profesores para la investigación, la enseñanza, el desarrollo del profesor y la mejora de la escuela. En C. M. Marcello, P. (Ed.), Pensamiento de los profesores y desarrollo profesional (pp. 203-219). Sevilla: Universidad de Sevilla.

Cain, T. (2005). Lo que aprendí de Alan: Un caso de cambio en la formación inicial del profesorado. Revista Electrónica Complutense de Investigación en Educación Musical, 2(4), 1-5. Consultado en http://revistas.ucm.es/index.php/RECI/article/view/RECI0505110004A 
Cantón, I. (1996). Los documentos personales al servicio de la investigación biográfica. En E. López-Barajas (Ed.), Las historias de vida y la investigación biográfica. Fundamentos y metodología (pp. 215-225). Madrid: Universidad Nacional de Educación a Distancia.

Cañada, M. (2008). Perfiles y profesiones de la música. Musiker: Cuadernos de Música, 16, 401406.

Colwell, R. (2007). Interlude: History looking forward. En Bresler (Ed.), International handbook of research in arts education (Vol. 1). Nueva York: Springer.

Cox, G. (2006). La investigación histórica en educación musical: Influencias de las ideas sobre la infancia, de las iglesias y de las escuelas. Revista Electrónica Complutense de Investigación en Educación Musical, 3(1), 1-10. Consultado en http://revistas.ucm.es/index.php/RECI /article/view/RECI0606110001A

Denzin, N. K. (1970). The research act. Chicago: Aldine.

Dorsam, P. A. (2006). Giovanni Battista Cirri: Analyses of his Sei Sonate a violoncello o violino e basso op. XVI, 1780 with a guide to performance practice and a history of the composer and his music (Italy). Boston University.

Eisner, E. W. (1998). El ojo ilustrado: Indagación cualitativa y mejora de la práctica educativa. Barcelona: Paidós.

Fernández Cruz, M. (1998). Ciclos de vida de la enseñanza. Cuardernos de Pedagogía, 266, 52-57.

Gagnon, M.-E. (2006). The influence of the French cello school in North America. Tesis doctoral inédita. University of Miami, Miami.

Hallam, S. (1998). Instrumental teaching: A practical guide to better teaching and learning. Oxford: Heinemann.

Hookey, M. (2001). Professional development. En R. R. Colwell, C. P (Ed.), The new handbook of music teaching and learning (pp. 887-904). Nueva York: Oxford University Press.

Jørgensen, H., \& Lehmann, A. C. (Ed.). (1997). Does practice make perfect? Current theory and research on instrumental music practice. Oslo: NMH.

Kelchtermans, G. (1993). Getting the story, understanding the lives: From career stories to teachers' professional development. Teaching and Teacher Education, 9(5/6), 443-456. doi: 10.1016/0742-051X(93)90029-G

Kelchtermans, G., \& Vandenberghe, R. (1994). Teachers' professional development: A biographical perspective. Journal Curriculum Studies, 26(1), 45-62.

Knowels, J. G. (2004). Modelos para la comprensión de las biografías del profesorado en formación y en sus primeros años de docencia. Ilustraciones a partir de estudios de caso. En I. F.Goodson (Ed.), Historias de vida del profesorado (pp. 149-205). Barcelona: Octaedro.

Laffite, R. (1991). Evaluación y desarrollo profesional del docente universitario: Dos facetas de la mejora institucional. Comunicación. III Jornadas de Didáctica Universitaria. .

Miles, M. B., \& Huberman, A. M. (1994). Qualitative data analysis: An expanded sourcebook. Thousand Oaks (California): Sage.

Ocaña, A. (2006a). Desarrollo profesional de las maestras de educación musical desde una perspectiva biográfico-narrativa. Revista Electrónica Complutense de Investigación en Educación Musical, 3(3), 1-14. Consultado en http://revistas.ucm.es/index.php/RECI /article/view/RECI0606110003A

Ocaña, A. (2006b). Identidad y ciclos de desarrollo profesional de los maestros y maestras de educación musical. Tesis doctoral inédita. Universidad de Granada, Granada. 
Park, H. Y. (2004). Laszlo Varga: Pedagogy, history, and legacy. Tesis doctoral inédita. University of Miami.

Pezzolli, G. A. (2011). William Henry Squire's out-of-print works for cello and piano: Analysis and suggestions for teachers. Tesis doctoral inédita. The University of North Carolina, Greensboro.

Polo, A. (2008). Los retos de la formación superior de violoncello en el País Vasco. Musiker: Cuadernos de Música, 16, 377-383.

Pujadas, J. J. (1992). El método biográfico: El uso de las historias de vida en Ciencias Sociales. Madrid: Centro de Investigaciones Sociológicas.

Rink, J. (1995). The practise of performance. Cambridge: Cambridge University Press.

Rink, J. (2002). Musical performance. Cambridge: Cambridge University Press.

Rusinek, G. (2004). Aprendizaje musical significativo. Revista Electrónica Complutense de Investigación en Educación Musical, 1(5). Consusltado en http://revistas.ucm.es/index.php /RECI/article/view/RECI0404110005A

Rusinek, G. (2006). Investigar en educación musical. Doce Notas, 53, 12-13.

Stake, R. (1998). Investigación con estudio de casos. Madrid: Morata.

Stowell, R. (Ed.). (1999). The Cambridge companion to the cello. Cambridge: Cambridge University Press.

Walden, V. (1998). One hundred years of violoncello. A history of technique and performance practice, 1740-1840. Cambridge: Cambridge University Press. 